\title{
High resolution observations of the hot core in G29.96-0.02
}

\author{
L. Olmi ${ }^{1,2}$, R. Cesaroni ${ }^{3}$, P. Hofner ${ }^{1,4}$, S. Kurtz ${ }^{6}$, E. Churchwell ${ }^{5}$, and C. M. Walmsley ${ }^{3}$ \\ 1 University of Puerto Rico, Physics Department, PO Box 23343 University Station, San Juan, PR 00931-3343, USA \\ 2 Istituto di Radio Astronomia, CNR Sezione di Firenze, Largo E. Fermi 5, 50125 Firenze, Italy \\ 3 Osservatorio Astrofisico di Arcetri, INAF, Largo E. Fermi 5, 50125 Firenze, Italy \\ e-mail: cesa@arcetri.astro.it; walmsley@arcetri.astro.it \\ 4 Arecibo Observatory, Cornell University, HC3 Box 53995, Arecibo, PR 00612, USA \\ e-mail: hofner@naic.edu \\ 5 Washburn Observatory, University of Wisconsin-Madison, 475 North Charter Street, Madison, WI 53706, USA \\ e-mail: ebc@astro.wisc.edu \\ ${ }^{6}$ Instituto de Astronomia, Universidad Nacional Autónoma de México, Apdo. Postal 3-72, C.P. 58090, Morelia, \\ Michoacán, Mexico \\ e-mail: s.kurtz@astrosmo.unam.mx
}

Received 12 February 2003 / Accepted 21 May 2003

\begin{abstract}
We present high angular resolution observations obtained with the Owens Valley and the IRAM Plateau de Bure millimeter-wave interferometers toward the hot core in $\mathrm{G} 29.96-0.02$. We observed the ground state $\mathrm{CH}_{3} \mathrm{CN}(6-5)$, $\mathrm{CH}_{3}^{13} \mathrm{CN}(6-5)$, vibrationally excited $\left(v_{8}=1\right) \mathrm{CH}_{3} \mathrm{CN}(6-5)$, and the $\mathrm{C}^{18} \mathrm{O}(1-0)$ rotational transitions, as well as the $2.7 \mathrm{~mm}$ continuum emission. Our continuum maps show evidence of a compact source barely resolved whose diameter we estimate to be about $0.06 \mathrm{pc}$ and whose emission mechanism is dominated by thermal emission from warm dust. Both the ground state and the $v_{8}=1$ methyl cyanide lines, as well as other serendipituosly detected molecular transitions, arise from a compact source at the same position as the $2.7 \mathrm{~mm}$ continuum emission. The $\mathrm{C}^{18} \mathrm{O}$ observations sample the structure and kinematics of the molecular surroundings of the hot core and from the $\mathrm{C}^{18} \mathrm{O}$ data we estimate a gas mass of about $1.1 \times 10^{3} M_{\odot}$ in a region with a diameter of $0.32 \mathrm{pc}$, corresponding to an average number density of about $10^{6} \mathrm{~cm}^{-3}$. Our data show evidence of both a temperature and density gradient in the hot core and its molecular surroundings. The density gradient, in particular, is consistent with the infalling scenario suggested by the presence of an East-West oriented velocity gradient, which is however of opposite sign in $\mathrm{CH}_{3} \mathrm{CN}$ and $\mathrm{C}^{18} \mathrm{O}$. We tentatively interpret the $\mathrm{C}^{18} \mathrm{O}$ velocity gradient as associated with infall, whereas the $\mathrm{CH}_{3} \mathrm{CN}$ gradient, consistent with that measured in $\mathrm{NH}_{3}$ by Cesaroni et al. (1998), is likely to trace a massive rotating disk.
\end{abstract}

Key words. stars: formation - radio lines: ISM - ISM: individual objects: G29.96-0.02 - ISM: molecules

\section{Introduction}

Ultracompact (UC) HII regions are used to trace high-mass star formation, and represent a relatively advanced evolutionary stage of a massive star. In fact, the earliest phases of evolution of a massive (proto)star are likely marked by the presence of dense, compact, and hot molecular cores (herafter HCs) surrounding the newly formed star. Such regions have been studied by several authors in different tracers and using different instruments (see Kurtz et al. 2000 for a review). However, all known HCs but Orion-KL lie at a distance between $\sim 2$ and $\sim 8.5 \mathrm{kpc}$ : this makes it difficult to determine their physical parameters without using high angular resolution, i.e. interferometric, observations. Furthermore, in order to study the earliest and most elusive evolutionary phase of main accretion, targets must be carefully selected. The approach used by Cesaroni et al. (1994b; hereafter CCHWK), consisted in searching for

Send offprint requests to: L. Olmi, e-mail: olmi@naic . edu massive protostars close to very young zero age main sequence (ZAMS) early-type stars (UC HII regions, in their case). This method is based on the fact that O-B stars form in clusters and hence it is likely to find high-mass protostars in association with slightly more evolved (but easier to identify) stars of the same type. Indeed, the study of CCHWK led to the identification of HCs close to, but generally not coincident with, UC HII regions.

G29.96-0.02 (hereafter G29) is an UC HII region located at a distance of $6 \mathrm{kpc}$ (Pratap et al. 1999; hereafter PMB) from the Sun. The UC HII region shows a clear cometary morphology (see e.g. Wood \& Churchwell 1989) and is embedded in a molecular cloud extensively studied at both low (Churchwell et al. 1990; Cesaroni et al. 1991, 1992; Churchwell et al. 1992) and high (CCHWK; Cesaroni et al. 1998, hereafter CHWC; Maxia et al. 2001; PMB) spatial resolution. In particular, CCHWK identified a HC lying in front of the cometary UC HII region, approximately 2.' 6 west of the $1.3 \mathrm{~cm}$ 
continuum peak; they also suggested that such a core is heated by a deeply embedded newly formed massive (proto)star. Subsequently, CHWC found evidence for the core to be slightly flattened east-west and rotating about an axis oriented approximately north-south: this suggests that we might be dealing with a rotating, massive circumstellar disk. PMB studied the molecular gas surrounding G29 with the BIMA interferometer at moderate angular resolution $\left(\sim 10^{\prime \prime}\right)$, and found an unresolved knot of $\mathrm{CH}_{3} \mathrm{CN}$ emission coincident with the $\mathrm{HC}$ previously detected in the $\mathrm{VLA} \mathrm{NH}_{3}(4,4)$ maps of CCHWK. PMB also detected a compact core of $\mathrm{CH}_{3} \mathrm{OH}$ emission, $\sim 5^{\prime \prime}$ southwest of the $\mathrm{HC}$, which they speculated to be associated with a molecular outflow from the $\mathrm{HC}$ exciting source or with a lower mass star forming outside it. However, further high angular resolution interferometric observations at 3 and $1.3 \mathrm{~mm}$ of the $\mathrm{HCO}^{+}(1-0)$ and $\mathrm{SiO}(2-1) v=0$ lines by Maxia et al. (2001) could not detect any clear signature of outflowing gas at the location of the $\mathrm{CH}_{3} \mathrm{OH}$ core. Their observations also appear to confirm the presence of a rotating disk around a young accretive massive (proto)star and of an $\mathrm{SiO}$ jet perpendicular to it. However, the kinematics of the region turns out to be quite complex and suggestive of infall onto the HC from the molecular surroundings of the UC HII-HC complex. Recently, Gibb et al. (2002) have used BIMA to map the G29 region at $1.4 \mathrm{~mm}$ in the continuum and a few molecular lines, among which $\mathrm{C}^{18} \mathrm{O}(2-1)$ and $\mathrm{H}_{2} \mathrm{~S} 2_{2,0}-2_{1,0}$. The most intriguing result is the detection in the $\mathrm{H}_{2} \mathrm{~S}$ line of a bipolar outflow arising from the $\mathrm{HC}$ : the flow axis is roughly perpendicular to the plane of the putative rotating disk observed by $\mathrm{CHWC}$ in ammonia thus leading support to the existence of such a disk.

In order to probe the detailed structure and kinematics of the HC we have used the IRAM-Plateau de Bure and the Owens Valley Radio Observatory interferometers to perform high angular resolution observations of $\mathrm{C}^{18} \mathrm{O}(1-0)$ and of $\mathrm{CH}_{3} \mathrm{CN}(6-5)$ and its isotopomer $\mathrm{CH}_{3}^{13} \mathrm{CN}(6-5)$, which are expected to provide information on the distribution, kinematics, and energetics of the molecular gas associated with the $\mathrm{HC}$, as demonstrated by analogous studies of similar objects (Cesaroni et al. 1994a; Olmi et al. 1996; Hofner et al. 1996; Zhang et al. 1998). We present our millimeter-wave interferometric observations and data reduction in Sect. 2. Our results are illustrated in Sect. 3, while in Sect. 4 we discuss the implications on the structure and nature of the $\mathrm{HC}$ and surrounding clump. Finally, the conclusions are drawn in Sect. 5.

\section{Observations}

\subsection{Owens Valley observations}

Observations of the $\mathrm{CH}_{3} \mathrm{CN}\left(J=6_{K}-5_{K}\right) K=0-5$ and $\mathrm{C}^{18} \mathrm{O}(J=1-0)$ spectral lines and of the $2.7 \mathrm{~mm}$ continuum were made between February and April 1995 using the Owens Valley millimeter-wave array (OVRO). The phase tracking center of the observations was $\alpha(\mathrm{J} 2000)=18^{\mathrm{h}} 46^{\mathrm{m}} 03^{\mathrm{s}} .955$, $\delta(\mathrm{J} 2000)=-02^{\circ} 39^{\prime} 21^{\prime \prime} .47$. We obtained data in three configurations with six antennas, using baselines between 15 and $240 \mathrm{~m}$.
We assumed rest frequencies of $110.383522 \mathrm{GHz}$ for $\mathrm{CH}_{3} \mathrm{CN}(\mathrm{J}=6-5) \mathrm{K}=0$ and $109.782160 \mathrm{GHz}$ for $\mathrm{C}^{18} \mathrm{O}(\mathrm{J}=$ 1-0) (Lovas 1992). Both lines were observed simultaneously in the lower sideband with a velocity resolution of $2.7 \mathrm{~km} \mathrm{~s}^{-1}$. Three 32-channel modules of the digital correlator were placed contiguously in frequency to cover the $K=0$ to $K=5$ components of the $J=6-5$ rotational transition of $\mathrm{CH}_{3} \mathrm{CN}$, resulting in a total bandwidth of $90 \mathrm{MHz}$ corresponding to a velocity coverage of $230 \mathrm{~km} \mathrm{~s}^{-1}$. The remaining 32 channel module was centered on the $\mathrm{C}^{18} \mathrm{O}(J=1-0)$ line providing a velocity range of about $80 \mathrm{~km} \mathrm{~s}^{-1}$. Both bands were centered at $V_{\mathrm{LSR}}=98.0 \mathrm{~km} \mathrm{~s}^{-1}$. A continuum measurement was obtained simultaneously with a bandwidth of $1 \mathrm{GHz}$.

We observed the quasar $1741-038$ every 25 minutes to calibrate visibility phase and gain variations. The absolute flux density scale was set by observations of Uranus (Ulich 1981) and 3C273. We estimate an amplitude uncertainty of about $10 \%$ and an absolute positional accuracy of about $0 .{ }^{\prime \prime} 3$. Observations of $3 \mathrm{C} 273$ were also used to derive bandpass corrections to the data.

After initial editing and calibration using the OVRO MMA software (Scoville et al. 1993), the data were imported into the NRAO data reduction package AIPS. We used the task IMAGR to create spectral line and continuum maps of size $512 \times 512$ pixels with 0 .' 3 per pixel using a variety of weighting schemes and range of UV data points. Depending on the weighting scheme and UV range used, we obtained

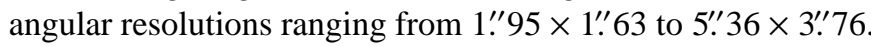
Line free channels were averaged and subsequently subtracted to form data cubes containing the spectral lines only.

\subsection{Plateau de Bure observations}

We used the IRAM ${ }^{1}$ interferometer at Plateau de Bure (PdBI) in the period February 1995 to April 1996. Observations of the $J=6-5$ rotational transition of $\mathrm{CH}_{3} \mathrm{CN}$ and $\mathrm{CH}_{3}^{13} \mathrm{CN}$ as well as several rotational transitions of vibrationally excited $\left(v_{8}=1\right)$ $\mathrm{CH}_{3} \mathrm{CN}$ were obtained in one configuration using 3 antennas and 4 configurations using 4 antennas, providing a total baseline coverage from 24 to $288 \mathrm{~m}$.

The phase tracking centre of the observations was $\alpha(\mathrm{J} 2000)=18^{\mathrm{h}} 46^{\mathrm{m}} 03^{\mathrm{s}} .955, \delta(\mathrm{J} 2000)=-02^{\circ} 39^{\prime} 21^{\prime \prime} .87$. All lines were observed simultaneously in the lower sideband and the receivers were tuned to $110.426 \mathrm{GHz}$ assuming an LSR velocity of $98.8 \mathrm{~km} \mathrm{~s}^{-1}$. The velocity resolution was $1.7 \mathrm{~km} \mathrm{~s}^{-1}$. We observed the various $K$-components of $\mathrm{CH}_{3} \mathrm{CN}(6-5)$ and $\mathrm{CH}_{3}{ }^{13} \mathrm{CN}(6-5)$ using two $80 \mathrm{MHz}$ sections of the spectral line correlator, which resulted in a total velocity coverage of about $350 \mathrm{~km} \mathrm{~s}^{-1}$. The same setup was used for observations of the vibrationally excited states.

Primary flux calibration as well as bandpass calibration was achieved by observations of 3 C273. Phase and gain calibrations were carried out using frequent observations of NRAO 530 and 1749+096 (B1950). The data were calibrated using the GILDAS software developed at IRAM and Observatoire

\footnotetext{
${ }^{1}$ IRAM is supported by INSU/CNRS (France), MPG (Germany) and IGN (Spain).
} 
de Grenoble. Images of size $128 \times 128$ pixels with a pixel size of 0 .' 3 per pixel were made and imported into AIPS for further analysis. The angular resolution obtained is of $3 . " 5 \times 1{ }^{\prime \prime} 4$.

\section{Results}

\subsection{Continuum emission}

In the two upper panels of Fig. 1, we show the $2.7 \mathrm{~mm}$ continuum map obtained with OVRO, while in the bottom panel the PdBI map is displayed. In order to achieve maximum sensitivity to extended structures, for the OVRO map we used natural weighting as well as a Gaussian taper to give lower weight to the long baseline data points (top panel). The beam of this image is $2{ }^{\prime \prime} 9 \times 22^{\prime \prime} 8$. The map is in excellent agreement with the $2 \mathrm{~cm}$ image of Fey et al. (1995; hereafter FGCV). In particular, many details of the extended emission behind the bright cometary arc can be seen in our map. One concludes that most of the observed flux at $3 \mathrm{~mm}$ is due to free-free emission from the UC HII region.

To achieve higher spatial resolution, we computed an image with uniform weighting, in which we also de-emphasized extended structures by omitting UV datapoints at spacings smaller than $20 \mathrm{k} \lambda$. The resulting image has a beam of 2 !" $0 \times 11^{\prime \prime} 6$ and is shown in the middle panel of Fig. 1. We see again a good reproduction of the cometary arc, known from previous observations at centimeter wavelengths, but in our $2.7 \mathrm{~mm}$ map a strong, fairly compact source is located in front of the arc. Deriving the observational parameters of this continuum source (hereafter referred to as the HC) is difficult due to extended emission in the region and to the proximity of the bright emission from the nearby UC HII region.

There are a number of high resolution images at $\mathrm{cm}$ wavelengths available for G29 (see below). None of these maps shows compact emission at the position of the HC. We thus assume that at $\mathrm{cm}$ wavelengths there is negligible contribution to the $\mathrm{HC}$ emission, and we use the $2 \mathrm{~cm}$ map of Wood \& Churchwell (1991) to subtract the unrelated diffuse continuum emission which contaminates the $\mathrm{HC}$ in the $2.7 \mathrm{~mm}$ map. After regridding and convolving the $2 \mathrm{~cm}$ map to the synthesized beam of the $2.7 \mathrm{~mm}$ data, we scaled the $2 \mathrm{~cm}$ image such that the peak position of the UC HII region has identical intensity in both images and subsequently subtracted this image from the $2.7 \mathrm{~mm}$ map. In this procedure we used the OVRO map because of its better UV coverage compared to the PdBI map. The resulting difference map is shown in Fig. 2. The bright UC HII emission near the $\mathrm{HC}$ has been removed in this map and the $\mathrm{HC}$ emission stands out clearly. Our method appears to have oversubtracted in the tail region of the UC HII region.

The peak position of the $\mathrm{HC}$ emission was determined by a 2D Gaussian fit using AIPS task JMFIT. The result is $\alpha(\mathrm{J} 2000)=18^{\mathrm{h}} 46^{\mathrm{m}} 03^{\mathrm{s}} .795, \delta(\mathrm{J} 2000)=-02^{\circ} 39^{\prime} 22^{\prime \prime} 08$, with an estimated positional uncertainty of $0 . \prime 3$ in each coordinate, and it is thus consistent with the peak position of the $\mathrm{NH}_{3}(4,4)$ emission measured by CCHWK with the VLA in its C-configuration. Our derived position of the HC is the same, within the positional uncertainty, as that shown in the higher resolution BIMA $1.4 \mathrm{~mm}$ continumm map of

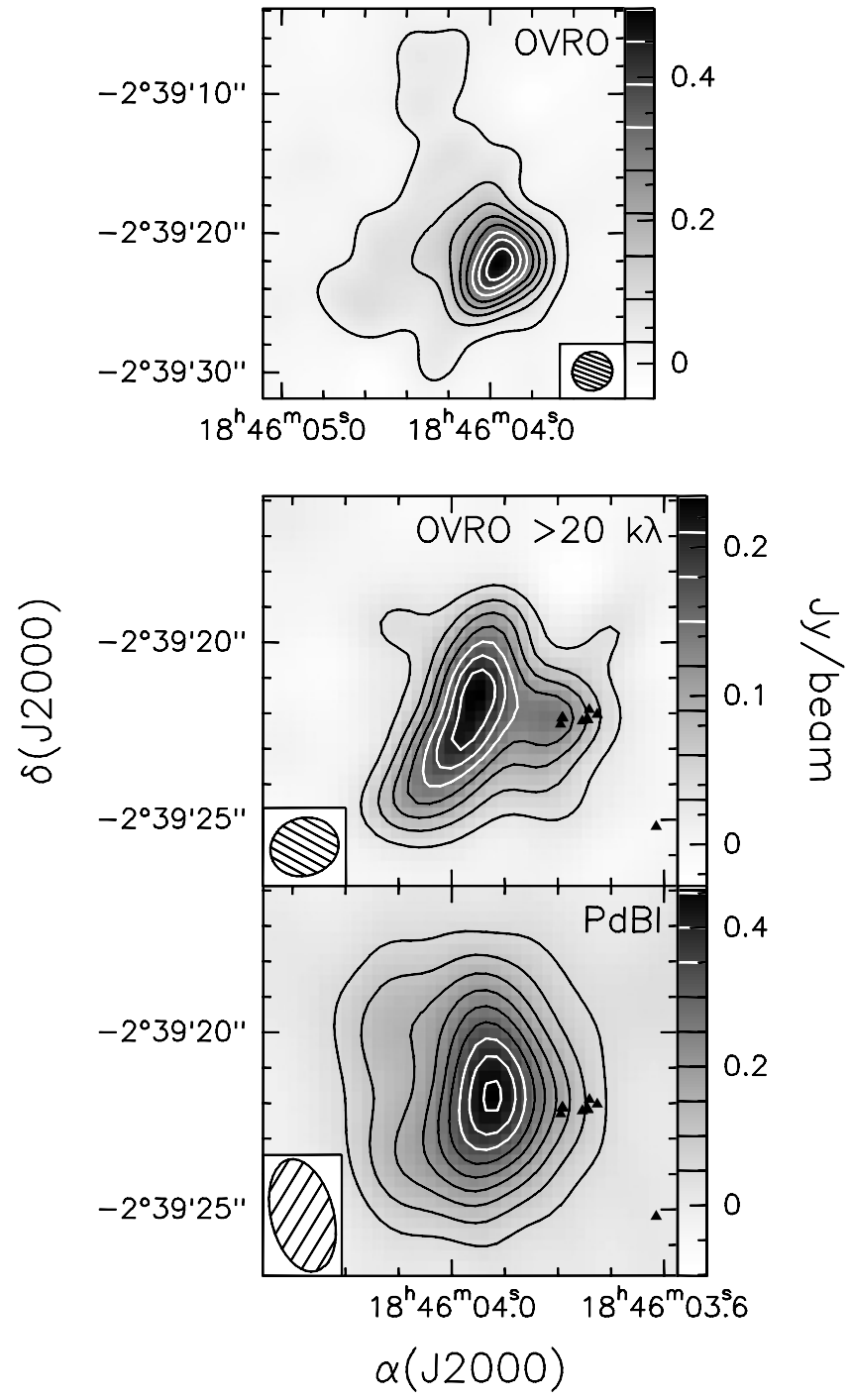

Fig. 1. Top: Map of the $2.7 \mathrm{~mm}$ continuum emission towards G29 obtained with OVRO. Natural weighting and Gaussian tapering has been used to enhance the extended structures. The synthesized beam is $2^{\prime \prime} 9 \times 2$.' 8 . Contour levels are drawn on the grey-scale wedge to the right of each panel. Middle: Same as top panel, but with uniform weighting and clipping of UV data points at spacings smaller than $20 \mathrm{k} \lambda$. The synthesized beam is $2^{\prime} \cdot 0 \times 1^{\prime \prime} 6$. The HC is visible about $2^{\prime \prime}$ West of the peak continuum emission. Bottom: Natural weighted map of the $2.7 \mathrm{~mm}$ continuum emission obtained with the PdBI. The triangles mark the positions of the $\mathrm{H}_{2} \mathrm{O}$ maser spots (Hofner \& Churchwell 1996). The synthesized beam is 3 '. $24 \times 1$ '. 77 with a position angle of $14^{\circ}$.

Wyrowski et al. (2002; hereafter WGM), confirming the reliability of the subtraction method described above. The HC emission appears fairly compact and unresolved in the east-west direction, but shows an extension oriented approximately north-south of FWHM 2."5, or about 1.'9 (0.055 pc) after deconvolution from the synthesized beam. This value is comparable to the deconvolved diameter (2." 3 ) obtained from the $1.4 \mathrm{~mm}$ map of WGM, whereas the size in the east-west direction is possibly affected by the subtraction process with the $2 \mathrm{~cm}$ map.

The integrated $2.7 \mathrm{~mm}$ continuum flux of the $\mathrm{HC}$ as measured in the difference map, is $150 \pm 50 \mathrm{mJy}$. The relatively large 


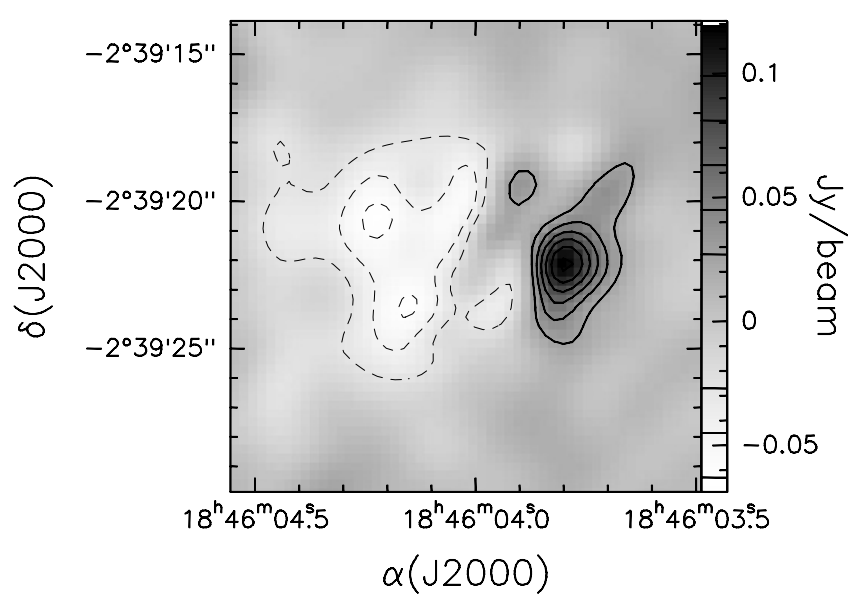

Fig. 2. Difference map obtained by subtracting the scaled $2 \mathrm{~cm}$ map of Wood \& Churchwell (1991) from the $2.7 \mathrm{~mm}$ OVRO map shown in the middle panel of Fig. 1 . The synthesized beam is 2 ! $9 \times 2$ '. 8 . Contour levels are drawn on the grey-scale wedge to the right.

assigned error in the total flux is dominated by uncertainties in the subtraction method, which are especially severe when one integrates over an area.

\subsubsection{The continuum spectrum of the hot core}

In order to perform a better analysis and derive the properties of the HC, in Fig. 3 we show the spectrum of the continuum emission from the HC. To attain a reliable comparison between fluxes measured at different wavelengths, all maps have been regridded and convolved to the beam of the $2.7 \mathrm{~mm}$ map and the emission has been integrated over an area of about $17 \operatorname{arcsec}^{2}$. This procedure has been repeated for the $20 \mathrm{~cm}$ continuum map of Claussen et al. (Priv. Comm.), the $6 \mathrm{~cm}$ continuum map of Afflerbach et al. (1994), the $2 \mathrm{~cm}$ continuum map of FGCV, the $1.3 \mathrm{~cm}$ continuum map of CCHWK, and the $1.4 \mathrm{~mm}$ continuum map of WGM. All of the above maps are of high quality and have been observed with the VLA or BIMA at a higher resolution than our $2.7 \mathrm{~mm}$ continuum map. In the same figure, also the 10 and $18 \mu \mathrm{m}$ measurements of De Buizer et al. (2002) and the mid- and far-IR fluxes of the corresponding IRAS and MSX counterparts are shown. It must be noted that the IRAS and MSX measurements are to be taken as upper limits to the corresponding $\mathrm{HC}$ fluxes, as the angular resolution is insufficient to separate the contribution of the $\mathrm{HC}$ from that of the nearby UC HII region.

In Fig. 3, one can see that at centimeter wavelengths the spectrum is dominated by free-free emission, which has been fitted (solid curve) with a spherical HII region with an angular diameter of 2 ". 1 and an emission measure of $3 \times 10^{7} \mathrm{pc} \mathrm{cm}^{-6}$. However, it must be stressed that such values do not correspond to a real HII region, but they rather represent a simple way to parametrise and fit the free-free measured towards the position of the HC. As a matter of fact, no clear continuum peak is detected towards the $\mathrm{HC}$ at centimeter wavelengths: therefore, the centimeter flux measured by us is to be attributed to diffuse free-free emission related to the nearby UC HII region.

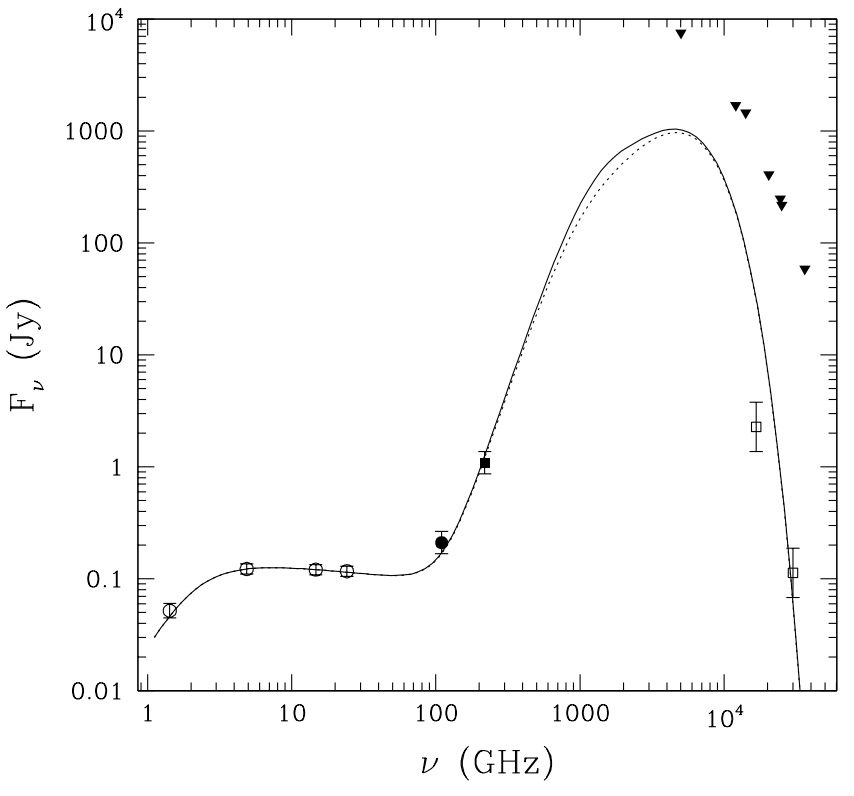

Fig. 3. Spectrum of the continuum emission measured towards the HC. The filled circle indicates the $2.7 \mathrm{~mm}$ flux obtained by us; the empty circles indicate the fluxes obtained at $20 \mathrm{~cm}, 6 \mathrm{~cm}, 2 \mathrm{~cm}$, and $1.3 \mathrm{~cm}$, after resampling and smoothing to the resolution of the $2.7 \mathrm{~mm}$ data the maps respectively of Claussen et al. (Priv. Comm.), Afflerbach et al. (1994), FGCV, and CCHWK; the filled square indicates the $1.4 \mathrm{~mm}$ flux of WGM; the empty squares are the fluxes measured by De Buizer et al. (2002) at 18 and $10 \mu \mathrm{m}$; and the filled triangles correspond to the fluxes of the IRAS and MSX point sources, and are to be taken as upper limits to the flux of the $\mathrm{HC}$ as the corresponding angular resolution is insufficient to resolve the core. The solid and dotted curves represent the fits obtained respectively with a spherical and a disk-like model (see text).

The HC, instead, is clearly identified in the millimeter and mid-IR images. After subtraction of the contribution from ionized gas, the spectral index between 2.7 and $1.4 \mathrm{~mm}$ is $\sim 4$ and hence consistent with optically thin, thermal emission from dust with $\beta \simeq 2$, where $\beta$ is the index of the dust opacity. The dust continuum emission can be fitted using a simple model based on a spherical core with constant density. We divide the core in spherical, concentric shells and solve the radiative transfer inside each shell assuming a constant temperature. The resulting emission is then used as background for the next shell. The temperature follows a power law, $T \propto R^{q}$, where $q=-2 /(4+\beta)$ (Larsson et al. 2000). The existence of such a gradient is indicated by the fact that different temperatures are measured at different radii by us and PMB. In our model we hence adopt the law $T=90 \mathrm{~K}\left(\theta / 1^{\prime \prime}\right)^{-1 / 3}$, with $\theta$ angular distance from the centre of the core. Following Kramer et al. (1998), we assume a total (dust+gas) absorption coefficient $\kappa(v)=0.005 \mathrm{~cm}^{2} \mathrm{~g}^{-1}(v / 231 \mathrm{GHz})^{\beta}$, with $\beta=2$. The free parameters of the model are the solid angle subtended by the core and the mass of the core. The former is estimated from the $1.4 \mathrm{~mm}$ continuum map of WGM, from which one derives a HC diameter of 2 '. 3 , after beam deconvolution. The best fit to the continuum spectrum (solid line in Fig. 3 ) is obtained for a mass of $320 M_{\odot}$. 


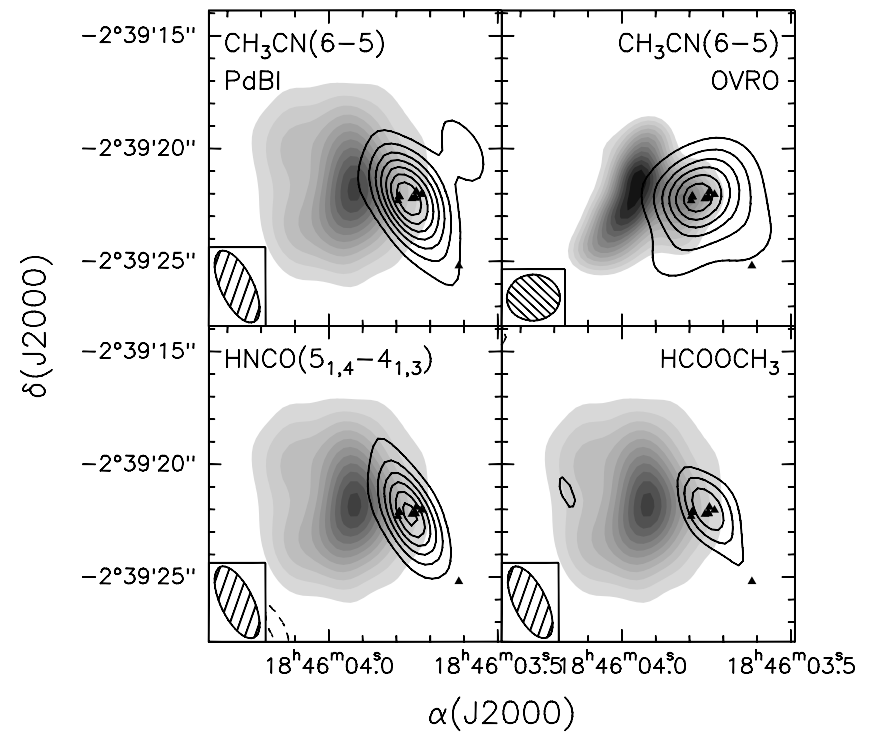

Fig. 4. Top panels: Maps of the $\mathrm{CH}_{3} \mathrm{CN}(6-5)$ emission averaged under the $K=0$ and 1 lines (contours) obtained with PdBI (left) and OVRO (right), overlaid on the corresponding $2.7 \mathrm{~mm}$ continuum image (grey scale). The OVRO continuum map is that given in the center panel of Fig. 1. Contour levels range from 0.12 to 0.84 in steps of $0.12 \mathrm{Jy} /$ beam for both $\mathrm{CH}_{3} \mathrm{CN}$ maps. Triangles mark the positions of the $\mathrm{H}_{2} \mathrm{O}$ maser spots (Hofner \& Churchwell 1996). Bottom: Maps of the emission averaged under the line for two molecular species serendipitously detected in the PdBI observations (contours) overlaid to the $2.7 \mathrm{~mm}$ continuum image (grey scale). Contour levels range from 0.05 to 0.3 in steps of $0.05 \mathrm{Jy} /$ beam for $\mathrm{HNCO}\left(5_{1,4}-4_{1,3}\right)$ and from 0.045 to 0.105 in steps of $0.02 \mathrm{Jy} /$ beam for the blend of $\mathrm{HCOOCH}_{3} \quad 9_{6,4}-8_{6,3} \mathrm{E}$, $9_{6,4}-8_{6,3} \mathrm{~A}$, and $9_{6,3}-8_{6,2} \mathrm{~A}$.

It is also interesting to consider the possibility that the $\mathrm{HC}$ is a disk-like structure, as suggested by CHWC on the basis of the temperature profile, $T \propto R^{-0.75}$, which is known to be typical of optically thick (at mid and far infrared wavelengths) passive and active disks (e.g., Pringle 1981). In fact, in Sect. 4.2 it will also be shown that the $\mathrm{CH}_{3} \mathrm{CN}$ lines arise from a region of $\sim 2^{\prime \prime}$ whereas the $\mathrm{CH}_{3}^{13} \mathrm{CN}$ and $v_{8}=1$ transitions trace a region of $\sim 1^{\prime \prime}$. Correspondingly, temperature estimates of $90 \mathrm{~K}$ from $\mathrm{CH}_{3} \mathrm{CN}$ (see $\mathrm{PMB}$ ) and $150 \mathrm{~K}$ from $\mathrm{CH}_{3}^{13} \mathrm{CN}$ and $v_{8}=1$ (see Fig. 8 ) have been obtained. This implies a slope $q \simeq-0.74$ for the temperature profile, in agreement with the value of -0.75 obtained by CHWC. Assuming that the disk is seen edge-on and using a similar model and the same input parameters as above, one obtains a best fit for a core mass of $260 M_{\odot}$ (dotted line in Fig. 3). Clearly the two fits are indistinguishable: both satisfy the data, with the sole exception of the $18 \mu \mathrm{m}$ flux measured by De Buizer et al. (2002). The latter, however, has been derived after subtracting the contribution of the nearby UC HII region and hence suffers by large uncertainties.

We conclude that our models provide satisfactory fits to the data point for a $\mathrm{HC}$ diameter of $0.067 \mathrm{pc}$, a surface temperature of $80 \mathrm{~K}$, a mass of 260-320 $M_{\odot}$ and, consequently, a density of $\sim 4 \times 10^{7} \mathrm{~cm}^{-3}$ and a column density of $8 \times 10^{24} \mathrm{~cm}^{-2}$. The luminosity of the $\mathrm{HC}$, estimated by integrating the emission under the fit to the continuum spectrum, is $\sim 9 \times 10^{4} L_{\odot}$, corresponding to an O7.5 ZAMS star (Panagia 1973).

\subsection{Methyl cyanide and other hot-core species}

Due to its high dipole moment of 3.91 debye, methyl cyanide is a good probe of densities $>10^{5} \mathrm{~cm}^{-3}$. The fact that all $K$-components of the $J=6-5$ rotational transition can be observed simultaneously allows under some circumstances an estimate of the gas temperature (see e.g. Olmi et al. 1993). Our expectation is that this molecule can probe the physical and kinematical structure of the HC in G29.

The distribution of the averaged $\mathrm{CH}_{3} \mathrm{CN}(J=6-5) \mathrm{K}=$ $0+1$ emission obtained with OVRO and PdBI is shown in the top panels of Fig. 4 as solid contours, superimposed on the grey-scale map of the corresponding $2.7 \mathrm{~mm}$ continuum emission. Likewise, the bottom panels in the same figure illustrate the distribution of the emission in a few serendipitously detected transitions of $\mathrm{HNCO}$ and $\mathrm{HCOOCH}_{3}$ : more precisely, we have detected the $9_{6,3}-8_{6,2} \mathrm{E}, 9_{6,4}-8_{6,3} \mathrm{E}, 9_{6,4}-$ $8_{6,3} \mathrm{~A}$, and $9_{6,3}-8_{6,2} \mathrm{~A}$ lines of $\mathrm{HCOOCH}_{3}$ and the $5_{1,4}-4_{1,3}$ line of HNCO. These are two well known HC species: for example, $\mathrm{HCOOCH}_{3}$ has been previously detected by Olmi et al. (1996) and Liu (2000) towards the HCs in G10.47+0.07 and $\mathrm{G} 31.41+0.31$. Therefore, as expected, their emission traces the same region as the $\mathrm{CH}_{3} \mathrm{CN}$ lines. Our $3 \mathrm{~mm} \mathrm{HNCO}$ observations complement the $1.4 \mathrm{~mm}$ observations of $\mathrm{HNCO} 10_{0,10^{-}}$ $9_{0,9}$ by Gibb et al. (2002) and confirm that towards G29 the emission in high-density, high-temperature tracers peaks on the $\mathrm{HC}$ itself. Note that the $v_{8}=1$ lines of $\mathrm{CH}_{3} \mathrm{CN}$ arise from approximately the same region as the ground state lines and we thus consider it superfluous to show the corresponding map here. Clearly, in all cases in Fig. 4 the emission looks at most barely resolved, although an estimate of the core diameter in the different transitions is possible. We shall come back to this point in Sect. 4.1.

It is also interesting to compare the $\mathrm{CH}_{3} \mathrm{CN}$ emission seen with the two interferometers to that measured with the 30-m telescope (Churchwell et al. 1992). This is done in Fig. 5, where we show the spectra obtained by integrating the $\mathrm{CH}_{3} \mathrm{CN}$ emission over the $\mathrm{HC}$ both for the OVRO and PdBI data. The horizontal thick bars represent the intensity of the $K$ lines derived from the spectrum in Fig. 3 of Churchwell et al. (1992). A minor difference $(\sim 10 \%)$ is found between the OVRO and PdBI spectra of the $K=0-3$ lines, which can be explained in terms of different UV coverages for the two instruments. In fact, the minimum OVRO baseline is half that of the most compact PdBI configuration used (see Sect. 2), and thus the OVRO observations are more sensitive to extended emission than the PdBI data. We also note that the difference between the OVRO and PdBI spectra is unlikely to be caused by a flux density calibration error, as the difference is not uniform across the spectrum, but it only affects the $K=0-3$ components.

The single-dish flux, instead, is consistent with the interferometric measurements only for the highest energy lines, i.e. for the $K=3-5$ components, whereas it is $\sim 1.5$ times stronger 


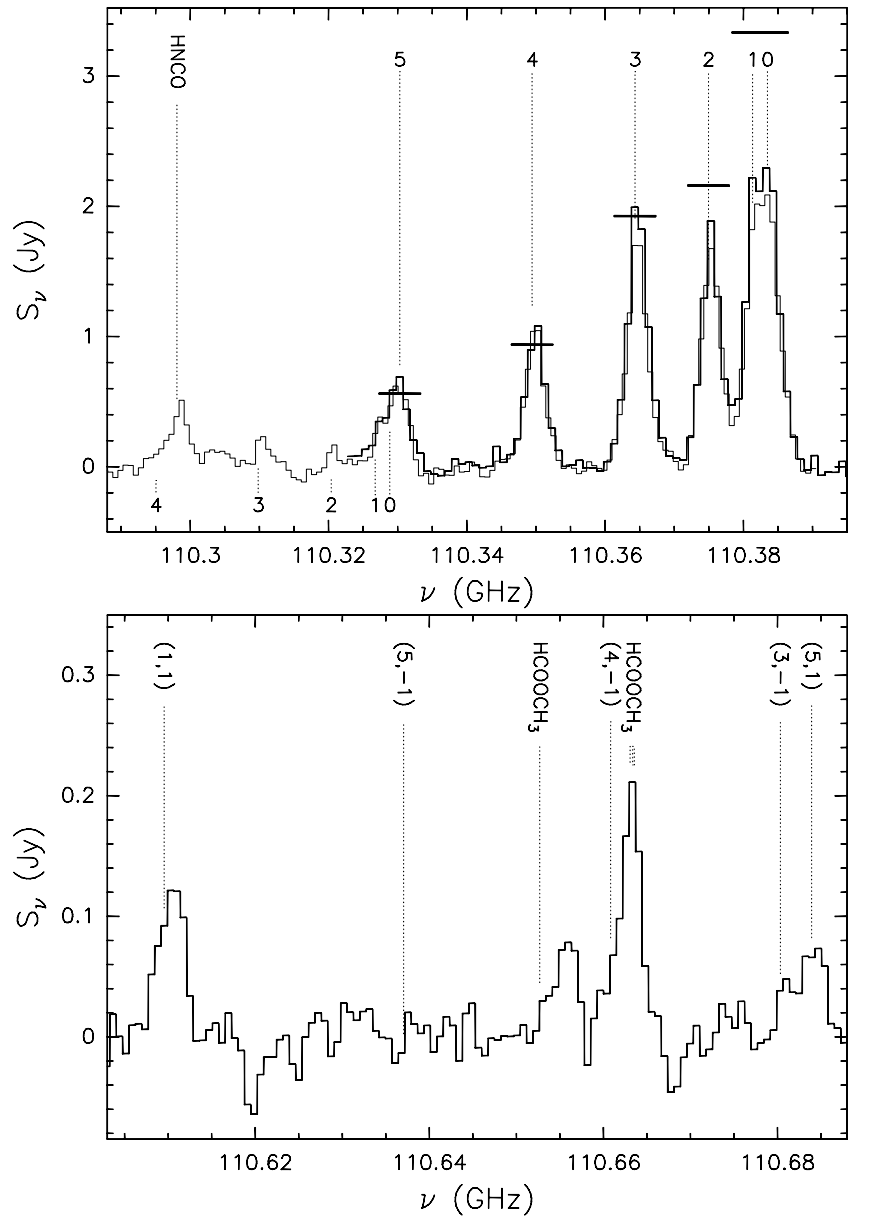

Fig. 5. Top: Comparison between the OVRO (full line) and PdBI (thin line) spectra obtained integrating over the area seen in the $\mathrm{CH}_{3} \mathrm{CN}(6-$ 5) $K=0+1$ line. The thick horizontal bars indicate the intensity measured with the IRAM 30-m telescope by Churchwell et al. (1992). The dotted vertical lines and corresponding labels indicate the positions of the $K$ components of the $\mathrm{CH}_{3} \mathrm{CN}(6-5)$ (top) and $\mathrm{CH}_{3}^{13} \mathrm{CN}(6-$ 5) (bottom) transitions, and that of the $\operatorname{HNCO}\left(5_{1,4}-4_{1,3}\right)$ line. Bottom: Integrated spectrum of the $v_{8}=1$ lines of $\mathrm{CH}_{3} \mathrm{CN}$ measured with the PdBI. The dotted vertical lines and corresponding labels mark the $(K, l)$ components of $\mathrm{CH}_{3} \mathrm{CN}$ and the $9_{6,3}-8_{6,2} \mathrm{~A}, 9_{6,4}-8_{6,3} \mathrm{~A}, 9_{6,4}-$ $8_{6,3}$ E, $9_{6,3}-8_{6,2}$ E transitions of $\mathrm{HCOOCH}_{3}$.

in the $K=0,1$ transitions. This demonstrates that part of the ground state $\mathrm{CH}_{3} \mathrm{CN}$ emission arises from an extended halo around the HC and UC HII complex: such a core-halo structure has been directly imaged in similar cases, like the HCs in G10.47+0.03 and G31.41+0.31 (Olmi et al. 1996).

\section{3. $\mathrm{C}^{18} \mathrm{O}$ emission}

In Fig. 6 we present a map of the $\mathrm{C}^{18} \mathrm{O}(1-0)$ emission averaged under the line, overlayed onto the corresponding $2.7 \mathrm{~mm}$ continuum map. The $\mathrm{C}^{18} \mathrm{O}$ map has an angular resolution $\sim 2.3$ times better than that achieved by PMB, which explains the different structure seen in our map with respect to their Fig. 6. While in the latter the emission is only barely resolved and seems to peak towards the UC HII region, our map traces also the $\mathrm{HC}$ - whose position is marked in Fig. 6

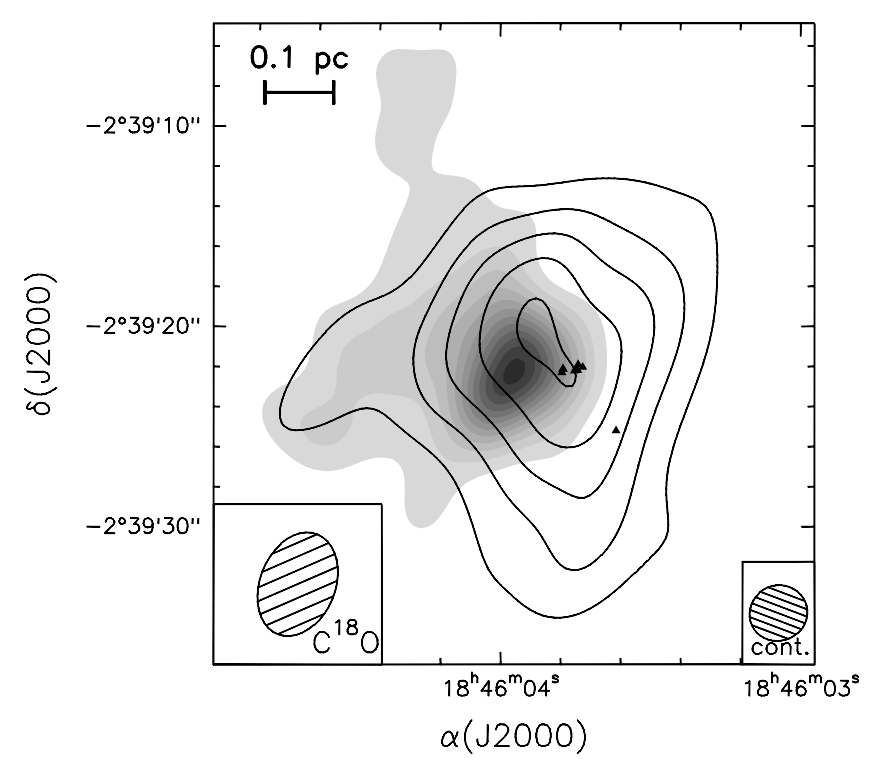

Fig. 6. Overlay of the $2.7 \mathrm{~mm}$ continuum image (grey scale) and $\mathrm{C}^{18} \mathrm{O}(1-0)$ line map (contours) observed with OVRO. The latter has been obtained averaging the emission from 95.28 to $100.74 \mathrm{~km} \mathrm{~s}^{-1}$. Contour levels range from 0.25 to 1.45 in steps of $0.3 \mathrm{Jy} / \mathrm{beam}$. Triangles mark the $\mathrm{H}_{2} \mathrm{O}$ maser spots observed by Hofner \& Churchwell (1996).

by the $\mathrm{H}_{2} \mathrm{O}$ maser spots. Also, one can see that most of the $\mathrm{C}^{18} \mathrm{O}$ emission is slightly offset from the UC HII region and seems to trace the dense gas around the cometary arc on a scale of $\sim 20^{\prime \prime}$ or $0.6 \mathrm{pc}$. We are obviously observing the densest portion of the cloud where the G29 region is embedded, as suggested by the fact that a large fraction of the flux emitted in the $\mathrm{C}^{18} \mathrm{O}(1-0)$ line is not imaged by the OVRO interferometer. This is demonstrated by Fig. 7, where a comparison is made between a single dish spectrum obtained towards G29 with the IRAM 30-m telescope (Lorenzani, Priv. Comm.) and the spectrum obtained from our OVRO data integrating the emission over the whole $\mathrm{C}^{18} \mathrm{O}$ clump shown in Fig. 6: clearly, approximately $50 \%$ of the flux is missing in the OVRO spectrum. In conclusion, we believe that our $\mathrm{C}^{18} \mathrm{O}$ observations represent a suitable tool to sample the structure and kinematics of the molecular surroundings of the UC HII region and HC. We delay a further discussion on this topic to Sect. 4.3.

\section{Discussion}

In this section we derive the physical parameters of the molecular gas and investigate their variation inside the molecular cloud.

\subsection{Mass and density}

The $\mathrm{C}^{18} \mathrm{O}$ emission can be used to measure the column density and hence the mass of the clump surrounding the UC HII region and the HC. These have been computed under the usual assumptions of LTE and optically thin emission in the $\mathrm{C}^{18} \mathrm{O}(1-0)$ line. We also assumed a $\mathrm{C}^{18} \mathrm{O}$ abundance of $2.7 \times 10^{-7}$ (Irvine et al. 1987; Wilson \& Rood 1994), 


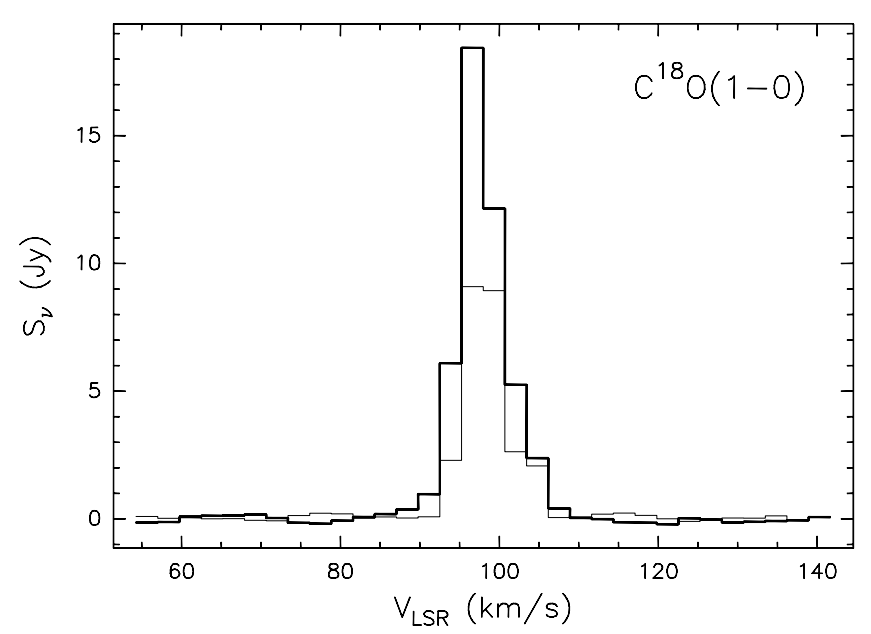

Fig. 7. Comparison between the OVRO (thin line) spectrum obtained integrating the $\mathrm{C}^{18} \mathrm{O}(1-0)$ line emission over the region in Fig. 6 and a single-dish spectrum (thick line) obtained with the IRAM 30-m telescope (Lorenzani, Priv. Comm.).

and a temperature ranging between $10 \mathrm{~K}$ (the peak brightness temperature of the $\mathrm{C}^{18} \mathrm{O}(1-0)$ line obtained after beam deconvolution and correcting the synthesized beam brightness temperature for the beam filling factor) and $150 \mathrm{~K}$ (the temperature of the $\mathrm{HC}$ derived from $\mathrm{CH}_{3} \mathrm{CN}$ - see below): the resulting mass ranges from 330 to $2900 M_{\odot}$. More likely, one can use the temperature estimate of $40 \mathrm{~K}$ obtained by PMB for the extended gas: in this case the mass is about $1190 M_{\odot}$, integrating the emission under the whole $\mathrm{C}^{18} \mathrm{O}$ line profile and over a total area of $342 \operatorname{arcsec}^{2}$ in the OVRO map. The corresponding virial mass can be calculated from the $\mathrm{C}^{18} \mathrm{O}(1-0)$ line width $\left(5.1 \mathrm{~km} \mathrm{~s}^{-1}\right)$ and diameter $(0.32 \mathrm{pc})$ and is equal to $870 M_{\odot}$, which is consistent with that calculated from the column density. The corresponding $\mathrm{H}_{2}$ density is $\sim 5 \times 10^{5} \mathrm{~cm}^{-3}$ for a deconvolved FWHM clump diameter of $11^{\prime \prime}$, i.e. 0.32 pc: the latter has been obtained after beam deconvolution from the full width at half power of the $\mathrm{C}^{18} \mathrm{O}(1-0)$ line map in Fig. 6 .

The previous mass estimate can be compared with the mass obtained from the single-dish $\mathrm{C}^{18} \mathrm{O}(1-0)$ spectrum shown in Fig. 7. By correcting for the different areas over which the emission has been integrated at the 30-m telescope (22" beam) and with OVRO (342 $\left.\operatorname{arcsec}^{2}\right)$, the resulting mass is about $1300 M_{\odot}$. Alternatively, we have also used the $\mathrm{C}^{18} \mathrm{O}(2-1)$ data of Hofner et al. (2000). Their measured integrated emission in the 11" beam of the IRAM 30-m telescope towards the $\mathrm{HC}$ position is $58 \mathrm{~K} \mathrm{~km} \mathrm{~s}^{-1}$ (in main beam temperature units) in the velocity interval 91 to $103 \mathrm{~km} \mathrm{~s}^{-1}$. Thus, the calculated gas mass is about $1070 M_{\odot}$, after scaling to the same integrating area av OVR and using the same $\mathrm{C}^{18} \mathrm{O}$ abundance and excitation temperature as above. Finally, we scaled the mass estimated by Hunter (1997), using the SHARC submillimeter wavelength data, to the same diameter (11 arcsec) and distance (6 kpc) as used here, obtaining a mass of about $2500 M_{\odot}$. Though larger than the previous estimates, it is still within the mass range $\left(330-2900 M_{\odot}\right)$ mentioned earlier.

All our mass estimates are thus consistent and the resulting number density can be used to calculate the pressure exerted by the molecular clump on the cometary UC HII region. Using an $\mathrm{H}_{2}$ density $n_{\mathrm{H}_{2}} \simeq 10^{6} \mathrm{~cm}^{-3}$ and the observed $\mathrm{C}^{18} \mathrm{O}(1-0)$ line FWHM $\left(\Delta v=5.1 \mathrm{~km} \mathrm{~s}^{-1}\right)$ mentioned above, the turbulent pressure of the molecular gas is $P_{\text {clump }}=n_{\mathrm{H}_{2}} \mu m_{\mathrm{H}} \Delta v^{2} /(8 \ln 2) \simeq$ $2.2 \times 10^{-7}$ dynes $\mathrm{cm}^{-2}$, where $m_{\mathrm{H}}$ is the mass of the hydrogen atom and $\mu=2.8$. We can compare this with the pressure of the ionized gas, $P_{\mathrm{UC}}=2 n_{\mathrm{e}} k T_{\mathrm{H}^{+}}+m_{\mathrm{H}} n_{\mathrm{e}} \Delta v_{\mathrm{UC}}^{2} /(8 \ln 2) \simeq$ $2.3 \times 10^{-7}$ dynes $\mathrm{cm}^{-2}$, where the values of the electron density, $n_{\mathrm{e}}=4.4 \times 10^{4} \mathrm{~cm}^{-3}$, and of the kinetic temperature, $T_{\mathrm{H}^{+}}=$ $6 \times 10^{3} \mathrm{~K}$, are both taken from Wood \& Churchwell (1991) and $\Delta v_{\mathrm{UC}} \simeq 35 \mathrm{~km} \mathrm{~s}^{-1}$ (PMB). Therefore, the turbulent pressure in the molecular gas appears large enough to confine the UC HII region, as also found by Olmi \& Cesaroni (1999).

Various density estimates for the molecular gas correspond to different scale sizes. In Sect. 3.1 we derived an $\mathrm{H}_{2}$ density of $4 \times 10^{7} \mathrm{~cm}^{-3}$ for the $0.067 \mathrm{pc}$ diameter $\mathrm{HC}$. The $\mathrm{C}^{18} \mathrm{O}$ analysis of this section yields a density of $9 \times 10^{5} \mathrm{~cm}^{-3}$ for a clump diameter of $11^{\prime \prime}(0.32 \mathrm{pc})$. PMB used the ${ }^{13} \mathrm{CO}(1-0)$ transition to obtain a density of $8 \times 10^{4} \mathrm{~cm}^{-3}$ over a region of $50^{\prime \prime}(1.5 \mathrm{pc})$. The trend of decreasing density with increasing size suggests a power law density gradient of approximately $n_{\mathrm{H}_{2}} \propto R^{-2}$, which is also consistent with the submillimeter wavelength data of Hunter (1997). Similar density gradients seem to be common in high-mass star forming regions (see Fontani et al. 2002) and suggest that the clumps containing newly formed massive stars are marginally stable. In fact, steep density profiles are expected in unstable configurations such as singular isothermal sphere (Shu et al. 1987; Li 1999). This result is in agreement with the infalling scenario proposed in Sect. 4.3.

If the clump is unstable, infall and mass accretion onto the embedded stars must occur. One may estimate the mass accretion rate by integrating the blue- and red-shifted $\mathrm{C}^{18} \mathrm{O}$ emission (see Fig. 12) over the corresponding region, resulting in a total accreting mass of $\sim 370 M_{\odot}$. The accretion time is given by the ratio between the size of the region $\left(\sim 11^{\prime \prime}\right.$ or $\left.0.32 \mathrm{pc}\right)$ and the maximum velocity (relative to the systemic LSR velocity of $98.9 \mathrm{~km} \mathrm{~s}^{-1}$ ) at which emission is detected in the $\mathrm{C}^{18} \mathrm{O}(1-0)$ line: this is $6 \times 10^{4} \mathrm{yr}$. Note that this value corresponds to the free-fall timescale, $t_{\mathrm{ff}}=(G \rho)^{-1 / 2} \simeq 6 \times 10^{4} \mathrm{yr}$, for $n_{\mathrm{H}_{2}}=10^{6} \mathrm{~cm}^{-3}$, approximately equal to the inferred density of the $\mathrm{C}^{18} \mathrm{O}$ clump. The accretion rate is hence $\dot{M} \simeq$ $6 \times 10^{-3} M_{\odot} \mathrm{yr}^{-1}$. This value is sufficient to quench the formation of an HII region in the HC even around an early $\mathrm{O}$ star (see Walmsley 1995), which could explain the existence of deeply embedded high-mass stars in the HC, even though no free-free emission has been detected towards it.

\subsection{Temperature}

The methyl cyanide data can also be used to determine the kinetic temperature, $T_{\text {kin }}$, and $\mathrm{CH}_{3} \mathrm{CN}$ column density of the HC. In Fig. 8 we show the rotation diagram obtained from the $\mathrm{CH}_{3} \mathrm{CN}(6-5)$ and $\mathrm{CH}_{3}^{13} \mathrm{CN}(6-5)$ ground state lines and the $\mathrm{CH}_{3} \mathrm{CN}(6-5)$ vibrationally excited transitions measured towards the centre of the HC. The ground state lines of the main species turn out to be optically thick $(\tau \simeq 10)$. Therefore, only the corresponding lines of the isotopomer have been used in the 


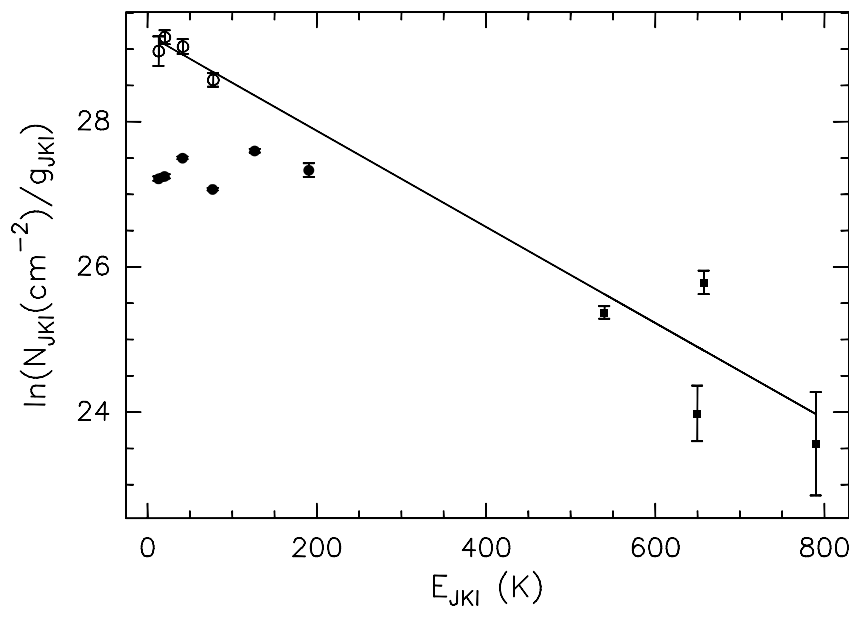

Fig. 8. Rotation diagram of the ground state (filled circles) and vibrationally excited (filled squares) lines of $\mathrm{CH}_{3} \mathrm{CN}(6-5)$, and ground state $\mathrm{CH}_{3}^{13} \mathrm{CN}(6-5)$ lines (empty circles), measured with the PdBI at the center position of the HC. The fit takes into account only the lines of the isotopomer and the vibrationally excited lines of the main species

fit after scaling their values by a relative abundance of 40 between $\mathrm{CH}_{3} \mathrm{CN}$ and $\mathrm{CH}_{3}^{13} \mathrm{CN}$. From Fig. 8 one derives a rotation temperature and total $\mathrm{CH}_{3} \mathrm{CN}$ column density $T_{\text {rot }} \simeq 150 \mathrm{~K}$ and $N_{\mathrm{CH}_{3} \mathrm{CN}} \simeq 3 \times 10^{16} \mathrm{~cm}^{-2}$. The former is considered a good approximation of $T_{\text {kin }}$ (see e.g. Olmi et al. 1993). We have then multipled $N_{\mathrm{CH}_{3} \mathrm{CN}}$ by the solid angle subtended by the $\mathrm{HC}$ to calculate the total number of $\mathrm{CH}_{3} \mathrm{CN}$ molecules in the $\mathrm{HC}$, and by comparing this to the $\mathrm{HC}$ mass obtained in Sect. 3.1 we have derived a $\mathrm{CH}_{3} \mathrm{CN}$ abundance relative to $\mathrm{H}_{2}$ of $\sim 5 \times 10^{-9}$.

Our value of $T_{\text {kin }} \simeq 150 \mathrm{~K}$ is significantly higher than the $90 \pm 30 \mathrm{~K}$ value computed by $\mathrm{PMB}$, also using $\mathrm{CH}_{3} \mathrm{CN}$. Very likely this is due to the fact that these authors have obtained their estimate from the ground state lines which may arise from a larger region than that traced by the $\mathrm{CH}_{3}^{13} \mathrm{CN}$ and $v_{8}=1$ transitions used in our derivation of the temperature. This suggests that the external layers of the HC are colder than the inner ones. The existence of a temperature gradient is confirmed by the analysis presented in Fig. 9, where we plot the deconvolved angular diameter of the $\mathrm{CH}_{3} \mathrm{CN}$ emission measured in each $K$ component, from which we see that the core is approximately 0.03 to $0.06 \mathrm{pc}$ in diameter, in agreement with the estimate given in Sect. 3.1. More importantly, this plot shows that higher energy transitions are excited closer to the centre, consistent with the idea of increasing temperatures toward the HC centre.

We note that optical depth effects could also explain the behaviour seen in Fig. 9. However, the same trend is seen in the $\mathrm{CH}_{3}^{13} \mathrm{CN}$ lines which are optically thin. Moreover, the diameter measured in the $(K, l)=(1,1)$ vibrationally excited line around $550 \mathrm{~K}$ is even smaller than that of the optically thin $\mathrm{CH}_{3}^{13} \mathrm{CN}$ transitions, thus demonstrating that the decrease of angular diameter cannot be due entirely to a decrease in optical depth.

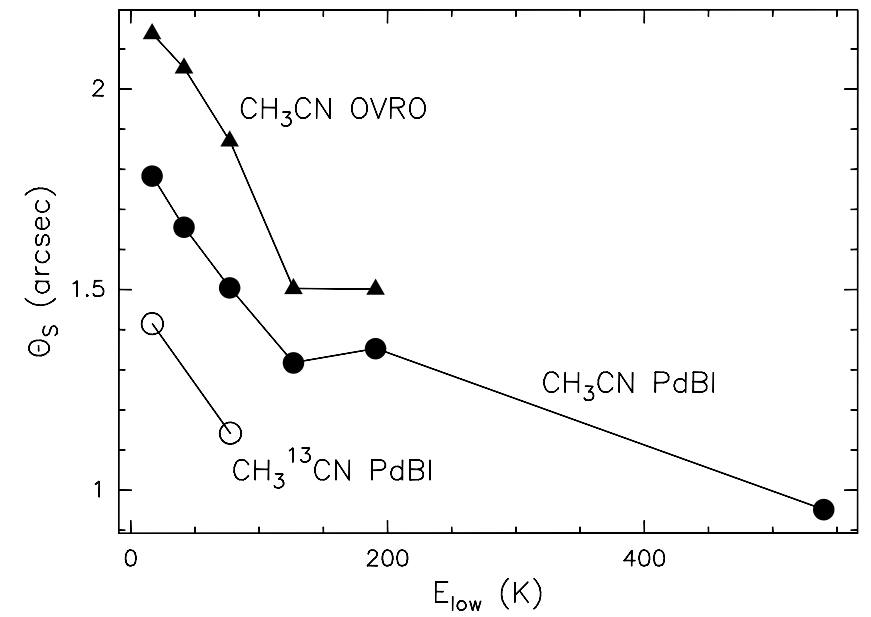

Fig. 9. Plot of the deconvolved angular diameter of the $\mathrm{HC}$ measured in the different lines of $\mathrm{CH}_{3} \mathrm{CN}$ (filled symbols) and $\mathrm{CH}_{3}^{13} \mathrm{CN}$ (empty symbols). Triangles and circles refer to the OVRO and PdBI data, respectively.

\subsection{Velocity gradient}

\subsection{1. $\mathrm{CH}_{3} \mathrm{CN}, \mathrm{C}^{18} \mathrm{O}$ and $\mathrm{NH}_{3}$ data}

To take full advantage of the velocity information contained in our $\mathrm{CH}_{3} \mathrm{CN}$ data, we fit a $2 \mathrm{D}$ Gaussian to the emission at each spectral channel. The results are shown in Fig. 10, where in the top panel we show a map with the distribution of the $\mathrm{CH}_{3} \mathrm{CN}$ peaks, obtained with the fitting procedure explained above. The positions of the $\mathrm{CH}_{3} \mathrm{CN}$ peaks observed with the OVRO interferometer define a straight line oriented approximately east-west. A velocity gradient is found along such direction and the best way to visualize it is to plot a position-velocity diagram along the RA axis, as shown in the bottom panel of Fig. 10. One can see that both the PdBI and OVRO data points present the same trend. The velocity gradient derived in this way is $\sim 25 \mathrm{~km} \mathrm{~s}^{-1} \operatorname{arcsec}^{-1}$ ( $\sim 860 \mathrm{~km} \mathrm{~s}^{-1} \mathrm{pc}^{-1}$ assuming a $6 \mathrm{kpc}$ distance to G29).

The velocity gradient observed in $\mathrm{CH}_{3} \mathrm{CN}$ has the same direction, though it is $\sim 10$ times larger, as that found in the $\mathrm{NH}_{3}(4,4)$ observations of CHWC, i.e. $2-3 \mathrm{~km} \mathrm{~s}^{-1} \operatorname{arcsec}^{-1}$ (after scaling to a distance of $6 \mathrm{kpc}$ ). The $\mathrm{CH}_{3} \mathrm{CN}$ velocity gradient is also larger than the value found from the $\mathrm{SiO}(2-1)$ observations of Maxia et al. (2001), i.e. $\sim 6 \mathrm{~km} \mathrm{~s}^{-1} \operatorname{arcsec}^{-1}$, though in this case the difference can be due to the fact that $\mathrm{SiO}$ is enhanced in shocked regions and may hence trace a different environment with respect to hot-core species like $\mathrm{CH}_{3} \mathrm{CN}$. On the other hand, one would expect to see the same velocity gradient in $\mathrm{CH}_{3} \mathrm{CN}$ and $\mathrm{NH}_{3}(4,4)$, since the high-excitation inversion transitions of ammonia are well known to trace HCs. However, the comparison between these two species may be affected by the different angular and spectral resolutions used, i.e. $\sim 2$." 2 and $\sim 2 \mathrm{~km} \mathrm{~s}^{-1}$ for $\mathrm{CH}_{3} \mathrm{CN}(6-5)$, and $\sim 0$ '. 35 and $\sim 5 \mathrm{~km} \mathrm{~s}^{-1}$ for $\mathrm{NH}_{3}(4,4)$. In order to demonstrate that such a discrepancy is due to the different methods used to estimate the velocity gradients in $\mathrm{CH}_{3} \mathrm{CN}$ and $\mathrm{NH}_{3}$, we show in Fig. 11 an overlay of the position-velocity diagrams for the $\mathrm{NH}_{3}$ data of CHWC and for the $\mathrm{PdBI} \mathrm{CH}{ }_{3} \mathrm{CN}$ data of this work. Clearly, the velocity 

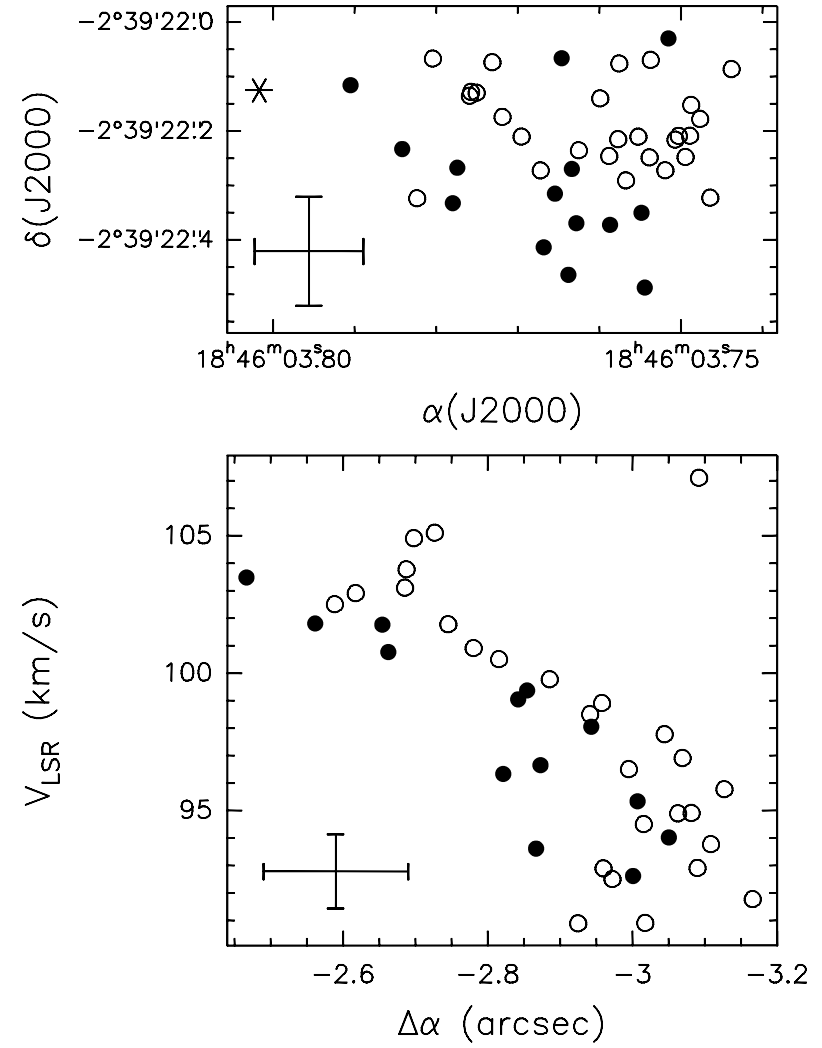

Fig. 10. Top: Distribution of the peak positions measured in different velocity channels for all the $\mathrm{CH}_{3} \mathrm{CN}(6-5) \mathrm{K}$ components. Filled and empty circles refer to OVRO and PdBI data, respectively. The asterisk marks the position of the dust continuum peak obtained by us as explained in Sect. 3.1. Bottom: Position-velocity plot along the RA axis obtained from the points in the top panel. Typical error bars are shown in the bottom left.

gradient derived from the $\mathrm{CH}_{3} \mathrm{CN}$ points (black dots in Fig. 11) is much greater than that obtained from the $\mathrm{NH}_{3}$ positionvelocity plot (dashed contours); however, the same positionvelocity plot for $\mathrm{CH}_{3} \mathrm{CN}$ (solid contours) seems consistent with that of $\mathrm{NH}_{3}$. We believe that different methods give different velocity gradients because of the higher spatial and lower spectral resolution of the $\mathrm{NH}_{3}$ data with respect to the $\mathrm{CH}_{3} \mathrm{CN}$ data. Presumably, if the $\mathrm{HC}$ were properly resolved both spatially and spectrally, the two estimates would match.

More intriguing is the difference between the velocity gradient seen in $\mathrm{CH}_{3} \mathrm{CN}$ and $\mathrm{C}^{18} \mathrm{O}$. In Fig. 12 we show an overlay of the $2.7 \mathrm{~mm}$ OVRO continuum map and the blue- and red-shifted $\mathrm{C}^{18} \mathrm{O}$ emission. Also shown are the $\mathrm{H}_{2} \mathrm{O}$ maser spots from Hofner \& Churchwell (1996): incidentally, we note that the isolated maser to the SW roughly coincides with the $\mathrm{CH}_{3} \mathrm{OH}$ peak found by $\mathrm{PMB}$, whereas the other masers cluster at the position of the HC. By comparing Figs. 10 and 12 one can see that while $\mathrm{C}^{18} \mathrm{O}$ traces a velocity gradient increasing from $\mathrm{E}$ to $\mathrm{W}$, the $\mathrm{CH}_{3} \mathrm{CN}$ velocity increases from $\mathrm{W}$ to $\mathrm{E}$, i.e. in the opposite direction. In the following we discuss various explanations for this opposite sense of direction of the velocity gradients.

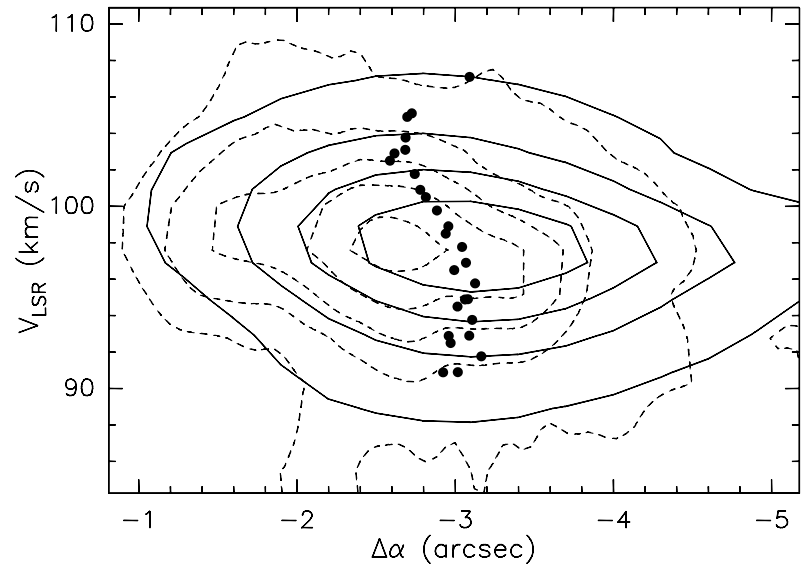

Fig. 11. Overlay of the PdBI $\mathrm{CH}_{3} \mathrm{CN}$ (solid contours) and $\mathrm{NH}_{3}$ (dashed contours) position-velocity diagram diagrams. Contour levels range from $10 \%$ to $90 \%$ in steps of $20 \%$ of the peak emission. The points are the same as in Fig. 10 for PdBI.

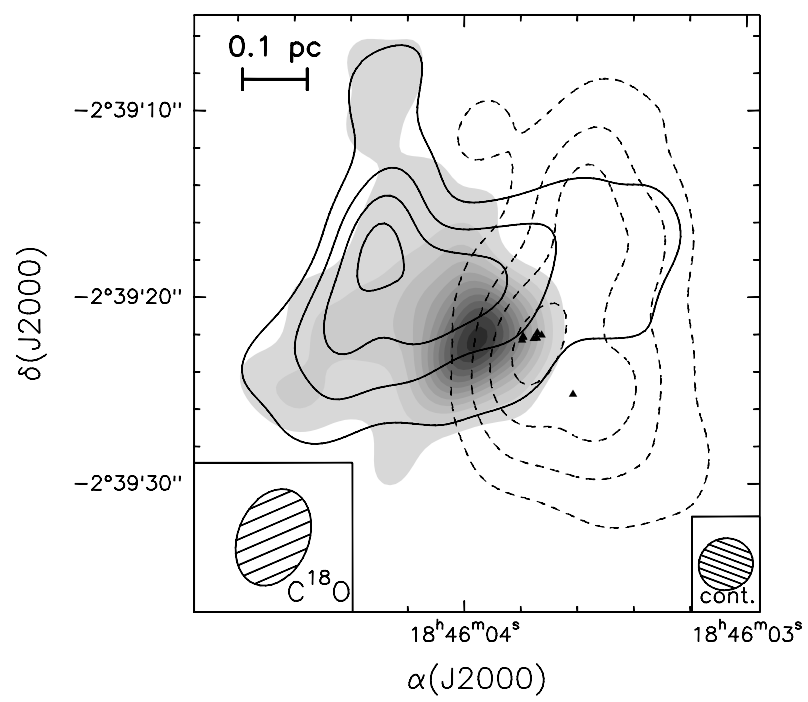

Fig. 12. Overlay of the $2.7 \mathrm{~mm}$ OVRO continuum map and the blue- (solid contours) and red-shifted (dashed contours) $\mathrm{C}^{18} \mathrm{O}$ emission. The latter have been obtained by integrating under the line wings over the velocity ranges $92.55-95.28 \mathrm{~km} \mathrm{~s}^{-1}$ and 100.74 $106.20 \mathrm{~km} \mathrm{~s}^{-1}$. Triangles mark the $\mathrm{H}_{2} \mathrm{O}$ maser spots observed by Hofner \& Churchwell (1996. Contour levels range from 0.11 to 0.44 in steps of $0.11 \mathrm{Jy} / \mathrm{beam}$.

\subsubsection{Possible interpretations of the $\mathrm{CH}_{3} \mathrm{CN}$ and $\mathrm{C}^{18} \mathrm{O}$ velocity fields}

In order to explain the velocity gradients observed in $\mathrm{CH}_{3} \mathrm{CN}$ and $\mathrm{C}^{18} \mathrm{O}$, one may basically think of three processes: infall, outflow, and rotation. While any of these can be invoked to explain the velocity gradient in just one species, the problem is to elaborate a scheme suitable to justify both tracers at the same time.

First of all, it is unlikely that the two velocity gradients are due to the same phenomenon. In fact, neither rotation nor infall can reverse the sign and the direction of the velocity even going from the large scale of $\mathrm{C}^{18} \mathrm{O}(\sim 0.6 \mathrm{pc})$ to the small $\mathrm{CH}_{3} \mathrm{CN}$ core $(\sim 0.06 \mathrm{pc})$. On the other hand, velocity reversals can be seen in 
outflows lying close to the plane of the sky (see e.g. Cesaroni et al. 1999) and precession of the outflow axis can change the direction of the velocity gradient on different spatial scales. However, a reason that advises against this scenario in G29 is that the velocity difference between the red- and blue-shifted emission of $\mathrm{C}^{18} \mathrm{O}$ and $\mathrm{CH}_{3} \mathrm{CN}$ is approximately the same, i.e. $\sim 15 \mathrm{~km} \mathrm{~s}^{-1}$, whereas one would expect the velocity range of $\mathrm{C}^{18} \mathrm{O}$ to be much broader because the latter traces a region which is about ten times larger than the $\mathrm{CH}_{3} \mathrm{CN}$ core. We also note that recently Gibb et al. (2002) have observed spatiallyresolved bipolar wing emission from $\mathrm{H}_{2} \mathrm{~S} 2_{2,0}-2_{1,0}$ in $\mathrm{G} 29$, centred on the $\mathrm{HC}$ and with an axis forming a large $\left(\gtrsim 50^{\circ}\right)$ angle with the direction of the $\mathrm{C}^{18} \mathrm{O}$ and $\mathrm{CH}_{3} \mathrm{CN}$ velocity gradients. This makes it unlikely that both $\mathrm{H}_{2} \mathrm{~S}$ and $\mathrm{C}^{18} \mathrm{O}$ trace an outflow.

Also unlikely is the possibility that $\mathrm{C}^{18} \mathrm{O}$ traces rotation and $\mathrm{CH}_{3} \mathrm{CN}$ an outflow, given the spatial scales involved in the two processes and the aforementioned results by Gibb et al. (2002). In the opposite case, if $\mathrm{C}^{18} \mathrm{O}$ were tracing an outflow and $\mathrm{CH}_{3} \mathrm{CN}$ a disk then the rotation axis of the disk should be aligned with the outflow axis and not roughly perpendicular to it. Another scenario which can be ruled out is that $\mathrm{CH}_{3} \mathrm{CN}$ is tracing infall. In fact, if the infall were occurring in a spherically symmetric environment, then one should see a blue-shifted self-absorption line profile because $\mathrm{CH}_{3} \mathrm{CN}$ is optically thick (see Sect. 4.2). However, this is ruled out by Fig. 5 where the unblended $K$ components of $\mathrm{CH}_{3} \mathrm{CN}$ have approximately Gaussian line profiles and do not present any hint of self-absorption nor any other peculiar asymmetry. If, on the other hand, the infall were occurring in a disk-like structure, which is non-rotating or where infall dominates over rotation, and which is inclined with respect to the line of sight, then the velocity gradient should be maximum along the apparent minor axis (i.e. along the declination, in our case) and not along the major axis, as shown in Fig. 10.

We thus think that the $\mathrm{C}^{18} \mathrm{O}$ gradient, rather than $\mathrm{CH}_{3} \mathrm{CN}$, is more likely associated with infall. Indeed, Maxia et al. (2001) have proposed a model where the large scale material is flowing along the surface of the UC HII region and collapsing towards the HC. Our results seem to confirm their interpretation. It is worth noting that in Fig. 12 the blue-shifted $\mathrm{C}^{18} \mathrm{O}$ emission matches very well the shape of the continuum emission, giving the impression that the molecular gas close to the surface of the HII region has been accelerated towards the observer. Albeit appealing, such an intepretation is ruled out by the results of Maxia et al. (2001), who have detected red-shifted absorption in the $\mathrm{HCO}^{+}(1-0)$ line in the direction of the HII region. This proves that the blue-shifted gas is not located between us and the HII region, but lies on the opposite side of it with respect to the observer. In other words, the blue-shifted gas is moving towards the HII region and not away from it. This is consistent with the Maxia et al. (2001) model where the large scale gas is collapsing towards the HC (see their Fig. 11 for a sketch of the model).

We still have to find an explanation for the velocity gradient seen in the $\mathrm{CH}_{3} \mathrm{CN}$, which is consistent with that found in the $\mathrm{NH}_{3}$ line. This cannot be associated with infall for the reasons explained above. Moreover, there is no reason why the sign of the infalling velocity should change from $\mathrm{C}^{18} \mathrm{O}$ to $\mathrm{CH}_{3} \mathrm{CN}$. On the other hand outflow cannot be excluded, although we consider it unlikely because the flow axis would be parallel to the direction along which infall seems to proceed on a large scale. More likely $\mathrm{CH}_{3} \mathrm{CN}$ is tracing a massive rotating disk as already found in other high-mass young stellar objects such as, e.g., G31.41+0.31 (Cesaroni et al. 1994a) and IRAS 20126+4104 (Cesaroni et al. 1999). Likewise, CHWC also found that a rotating disk in G29 is a suitable explanation for the velocity gradient measured in the $\mathrm{NH}_{3}$ lines. Further support to this interpretation is given by the bipolarity revealed by Gibb et al. (2002) in the red- and blueshifted $\mathrm{H}_{2} \mathrm{~S}$ line emission. This is intepreted by them as a bipolar outflow powered by a young stellar object embedded in the $\mathrm{HC}$. The flow axis is roughly perpendicular to the direction of the $\mathrm{NH}_{3}$ and $\mathrm{CH}_{3} \mathrm{CN}$ velocity gradient, as expected if the latter is observed along the plane of a rotating disk.

A final remark concerns the possibility that the $\mathrm{C}^{18} \mathrm{O}$ and $\mathrm{CH}_{3} \mathrm{CN}$ velocity gradients are related to outflows powered by two distinct young stellar objects. Obviously such an interpretation cannot be excluded, although this would imply the existence of two objects in quite a different evolutionary stage, being the kinematical age of the flows equal to $5 \times 10^{4} \mathrm{yr}$ for $\mathrm{C}^{18} \mathrm{O}$ and $2 \times 10^{3} \mathrm{yr}$ for $\mathrm{CH}_{3} \mathrm{CN}$.

\section{Conclusions}

We have used spectral line and continuum emission high angular resolution observations, obtained with the Owens Valley and the IRAM Plateau de Bure millimeter-wave interferometers, to probe the detailed structure and kinematics of the hot core identified by CCHWK and lying in front of the cometary UC HII region G29.96-0.02. Our $2.7 \mathrm{~mm}$ continuum maps show evidence of a compact source barely resolved whose diameter we estimate to be about $0.06 \mathrm{pc}$. The emission mechanism of this source is dominated by thermal emission from warm dust: the continuum spectrum from the millimeter to the mid-IR domain is fitted with a spherically symmetric or a disklike model with a temperature law $T \propto R^{-0.75}$. The best fit is obtained for a mass of $\sim 300 M_{\odot}$. The corresponding HC luminosity is $\sim 9 \times 10^{4} L_{\odot}$.

We have used the high dipole moment methyl cyanide molecule to probe the physical and kinematical structure of the HC. Both the ground state and the $v_{8}=1$ lines, as well as other serendipituosly detected molecular transitions, arise from a compact source at the same position as the $2.7 \mathrm{~mm}$ continuum emission and with a similar diameter. Comparing our interferometric data with existing single-dish observations we also find evidence that part of the ground state $\mathrm{CH}_{3} \mathrm{CN}$ emission arises from an extended halo around the hot core.

We have used the $\mathrm{C}^{18} \mathrm{O}$ observations to sample the structure and kinematics of the molecular surroundings of the UC HII region and of the hot core, and we have estimated a gas mass of about $1.1 \times 10^{3} M_{\odot}$ in a region with a diameter of $0.32 \mathrm{pc}$. The resulting number density of about $10^{6} \mathrm{~cm}^{-3}$ was used to calculate the turbulent pressure exerted by the molecular clump on the cometary UC HII region, which turns out to be large enough to confine the HII region. 
Using the density estimates from this work and those found in the literature we found that the data can be approximated by a power-law dependence on the radius of the type $n_{\mathrm{H}_{2}} \propto R^{-2}$, in the molecular surroundings of the hot core. The methyl cyanide data were also used to determine the kinetic temperature of the $\mathrm{HC}$, and by using the rotation diagram we derived a rotation temperature $T_{\text {rot }} \simeq T_{\text {kin }} \simeq 150 \mathrm{~K}$. The density gradient is consistent with the infalling scenario suggested by the presence of an East-West oriented velocity gradient.

Our high resolution observations provide new evidence and are consistent with earlier claims (CHWC) of the presence of a rotating disk around young accreting massive (proto-)star(s) located at the position of the hot core in the G29 region. The disk is roughly perpendicular to the axis of the bipolar outflow observed by Gibb et al. (2002). Our data also confirm that steep density and temperature gradients are common in high-mass star forming regions (see Fontani et al. 2002).

Acknowledgements. We acknowledge the IRAM staff from the Plateau de Bure for carrying out the observations. It is also a pleasure to thank R. Neri for his support during the PdBI data reduction, F. Wyrowski and A. Gibb for allowing us to use their BIMA $1.4 \mathrm{~mm}$ continuum map, and A. Fey for the $2 \mathrm{~cm}$ data. We also thank the referee, R. Liseau, for providing comments that improved this paper. P.H. acknowledges partial support from the Research Corporation Grant N. CC4996 and NSF grant AST-0098524.

\section{References}

Afflerbach, A., Churchwell, E., Hofner, P., \& Kurtz, S. 1994, ApJ, 437, 697

Cesaroni, R., Walmsley, C. M., Kömpe, C., \& Churchwell, E. 1991, A\&A, 252, 278

Cesaroni, R., Walmsley, C. M., \& Churchwell, E. 1992, A\&A, 256, 618

Cesaroni, R., Olmi, L., Walmsley, C. M., Churchwell, E., \& Hofner, P. 1994a, ApJ, 435, L137

Cesaroni, R., Churchwell, E., Hofner, P., Walmsley, C. M., \& Kurtz, S. 1994b, A\&A, 288, 903 (CCHWK)

Cesaroni, R., Churchwell, E., Hofner, P., \& Walmsley, C. M. 1998, A\&A, 331, 709 (CHWC)

Cesaroni, R., Felli, M., Jenness, T., et al. 1999, A\&A, 345, 949

Churchwell, E., Walmsley, C. M., \& Cesaroni, R. 1990, A\&AS, 83, 119

Churchwell, E., Walmsley, C. M., \& Wood, D. O. S. 1992, A\&A, 253, 541
De Buizer, J. M., Watson, A. M., Radomski, J. T., Piña, R. K., \& Telesco, C. M. 2003, ApJ, 564, L101

Fey, A. L., Gaume, R. A., Claussen, M. J., \& Vrba, F. J. 1995, ApJ, 453, 308

Fontani, F., Cesaroni, R., Caselli, P., \& Olmi, L. 2002, A\&A, 389, 603

Gibb, A. G., Wyrowski, F., \& Mundy, L. G. 2002, Chemistry as a Diagnostic of Star Formation, ed. C. L. Curry, \& M. Fich (University of Waterloo, Canada)

Hofner, P., \& Churchwell, E. 1996, A\&AS, 120, 283

Hofner, P., Kurtz, S., Churchwell, E., Walmsley, C. M., \& Cesaroni, R. 1996, ApJ, 460, 359

Hofner, P., Wyrowski, F., Walmsley, C. M., \& Churchwell, E. 2000, ApJ, 536, 393

Hunter, T. R. 1997, Ph.D. Thesis, California Institute of Technology

Irvine, W. M., Goldsmith, P. F., \& Hjalmarson, H. 1987, Interstellar Processes, ed. D. J. Hollenbach, \& H. A. Thronson, 561

Kramer, C., Alves, J., Lada, C., et al. 1998, A\&A, 329, L33

Kurtz, S., Cesaroni, R., Churchwell, E., Hofner, P., \& Walmsley, C. M. 2000, Protostars and Planets IV, ed. V. Mannings, A. Boss, \& S. Russel (Tucson: Univ. of Arizona Press)

Larsson, B., Liseau, R., Men'shchikov, A. B., et al. 2000, A\&A, 363, 253

Li, Z. 1999, ApJ, 526, 806

Liu, S.-Y., 2000, Am. Astron. Soc. Meet. 197, contribution, 132.01

Lovas, F. J. 1992, J. of Phys. and Chem. Ref. Data, 21, 181

Maxia, C., Testi, L., Cesaroni, R., \& Walmsley, C. M. 2001, A\&A, 371,287

Olmi, L., Cesaroni, R., \& Walmsley, C. M. 1993, A\&A, 276, 489

Olmi, L., Cesaroni, R., Neri, R., \& Walmsley, C. M. 1996, A\&A, 315, 565

Olmi, L., \& Cesaroni 1999, A\&A, 352, 266

Panagia, N. 1973, AJ, 78, 929

Pratap, P., Megeath, S. T., \& Bergin, E. A. 1999, ApJ, 517, 799 (PMB)

Pringle, J. E. 1981, ARA\&A, 19, 137

Scoville, N. Z., Carlstrom, J. E., Chandler, C. J., et al. 1993, PASP, 105,1482

Shu, F. H., Adams, F. C., \& Lizano, S. 1987, ARA\&A, 25, 23

Ulich, B. L. 1981, AJ, 86, 1619

Walmsley, C. M. 1995, Rev. Mex. Astron. Astrofis., Ser. de Conf., 1, 137

Wilson, T. L., \& Rood, R. 1994, ARA\&A, 32, 191

Wood, D. O. S., \& Churchwell, E. 1989, ApJS, 69, 831

Wood, D. O. S., \& Churchwell, E. 1991, ApJ, 372, 199

Wyrowski, F., Gibb, A. G., \& Mundy, L. G. 2002, Hot Star Workshop III: The Earliest Stages of Massive Star Birth, ASP Conf. Ser., ed. P. A. Crowther (WGM)

Zhang, Q., Ho, P. T. P., \& Ohashi, N. 1998, ApJ, 494, 636 